\title{
Erratum to: A hybrid discrete particle swarm optimization algorithm for solving fuzzy job shop scheduling problem
}

\author{
Jun-qing Li • Yu-xia Pan
}

Published online: 8 December 2012

(C) Springer-Verlag London 2012

\section{Erratum to: Int J Adv Manuf Technol}

\section{DOI 10.1007/S00170-012-43373}

The erratum for the paper IJAMT9306 (titled "A hybrid discrete particle swarm optimization algorithm for solving fuzzy job-shop scheduling problem"), which has been published online at 7/9/2012 (doi: 10.1007/s00170-012-4337-3).

Table 5 Numerical example Case5. (10-jobs-10-machines)

\begin{tabular}{|c|c|c|c|c|c|c|c|c|c|c|}
\hline \multicolumn{11}{|c|}{ Processing machines (fuzzy processing time) } \\
\hline Job1 & $8(2,3,4)$ & $6(3,5,6)$ & $5(2,4,5)$ & $2(4,5,6)$ & $1(1,2,3)$ & $3(3,5,6)$ & $9(2,3,5)$ & $4(1,2,3)$ & $7(3,4,5)$ & $10(2,3,4)$ \\
\hline Job2 & $10(2,3,4)$ & $7(2,3,5)$ & $4(2,4,5)$ & $6(1,2,3)$ & $8(4,5,6)$ & $3(2,4,6)$ & $2(2,3,4)$ & $1(1,3,4)$ & $5(2,3,4)$ & $9(3,4,5)$ \\
\hline Job3 & $6(2,4,5)$ & $9(1,2,3)$ & $10(2,3,5)$ & $8(1,2,4)$ & $1(3,5,6)$ & $7(1,3,4)$ & $4(1,3,5)$ & $2(1,2,4)$ & $5(2,4,5)$ & $3(1,3,5)$ \\
\hline Job4 & $1(1,2,3)$ & $5(3,4,5)$ & $8(1,3,5)$ & $9(2,4,6)$ & $10(2,4,5)$ & $6(1,2,4)$ & $7(3,4,5)$ & $2(1,3,5)$ & $4(1,3,6)$ & $3(1,3,4)$ \\
\hline Job5 & $2(2,3,4)$ & $7(1,3,4)$ & $3(1,3,4)$ & $5(1,2,3)$ & $8(1,3,5)$ & $9(2,3,4)$ & $10(3,4,5)$ & $6(1,3,4)$ & $1(3,4,5)$ & $4(1,3,4)$ \\
\hline Job6 & $4(2,3,4)$ & $2(2,3,4)$ & $3(1,2,3)$ & $5(2,4,5)$ & $6(1,3,4)$ & $8(1,3,4)$ & $7(3,4,5)$ & $9(1,2,3)$ & $10(2,4,5)$ & $1(1,3,4)$ \\
\hline Job7 & $3(2,3,4)$ & $5(1,4,5)$ & $4(1,3,5)$ & $1(3,4,5)$ & $9(2,3,4)$ & $7(3,4,5)$ & $2(1,2,3)$ & $10(3,5,6)$ & $8(3,5,6)$ & $6(1,2,3)$ \\
\hline Job8 & $7(3,4,5)$ & $1(1,2,3)$ & $9(3,4,5)$ & $6(2,4,5)$ & $10(1,3,4)$ & $2(2,3,4)$ & $5(1,2,3)$ & $3(2,4,5)$ & $4(3,4,5)$ & $8(2,3,5)$ \\
\hline Job9 & $9(3,4,5)$ & $4(1,3,4)$ & $10(1,3,5)$ & $2(2,3,4)$ & $3(3,5,6)$ & $6(2,4,5)$ & $8(1,3,4)$ & $1(3,4,5)$ & $5(1,2,3)$ & $7(3,4,5)$ \\
\hline Job10 & $7(2,4,5)$ & $5(1,2,3)$ & $2(3,4,5)$ & $4(2,3,4)$ & $1(1,2,3)$ & $8(3,4,5)$ & $10(2,4,5)$ & $6(3,4,5)$ & $3(1,2,3)$ & $9(1,2,4)$ \\
\hline
\end{tabular}

The online version of the original article can be found at http:// dx.doi.org/10.1007/s00170-012-4337-3.

J.-q. Li $(\bowtie) \cdot$ Y.-x. Pan

School of Computer Science, Liaocheng University, Liaocheng 252059, China

e-mail: p2p_jq1@126.com 
Table 7 Numerical example Case7. (10-jobs-10-machines)

Processing machines (fuzzy processing time)

\begin{tabular}{|c|c|c|c|c|c|c|c|c|c|c|}
\hline Job1 & $7(3,4,5)$ & $9(10,12,13)$ & $2(4,7,10)$ & $5(5,8,9)$ & $4(10,12,16)$ & $1(10,11,13)$ & $6(4,7,10)$ & $3(4,5,8)$ & $8(4,6,7)$ & $10(9,12,13)$ \\
\hline Job2 & $10(10,11,14)$ & $3(9,12,14)$ & $5(7,10,14)$ & $4(7,11,12)$ & $8(2,4,6)$ & $1(8,10,14)$ & $2(7,11,12)$ & $7(8,11,14)$ & $6(6,9,10)$ & $9(10,14,15)$ \\
\hline Job3 & $5(5,7,10)$ & $7(2,3,5)$ & $4(1,3,4)$ & $3(7,10,12)$ & $1(8,11,12)$ & $8(2,4,5)$ & $6(4,5,7)$ & $2(7,8,10)$ & $9(9,13,14)$ & $10(8,12,15)$ \\
\hline Job4 & $5(5,8,10)$ & $6(4,5,8)$ & $4(7,10,14)$ & $7(3,5,7)$ & $8(4,5,6)$ & $2(8,10,12)$ & $1(2,3,4)$ & $9(2,3,5)$ & $3(6,8,11)$ & $10(6,8,11)$ \\
\hline Job5 & $2(4,7,10)$ & $7(5,6,7)$ & $4(9,10,14)$ & $1(2,3,4)$ & $3(9,12,13)$ & $5(5,6,9)$ & $9(5,7,8)$ & $6(1,2,4)$ & $8(3,4,6)$ & $10(2,4,6)$ \\
\hline Job6 & $7(1,3,4)$ & $3(3,4,5)$ & $8(3,5,6)$ & $2(5,7,8)$ & $4(8,9,13)$ & $9(9,12,14)$ & $10(4,7,8)$ & $1(1,2,4)$ & $6(2,4,5)$ & $5(6,9,12)$ \\
\hline Job7 & $10(10,11,14)$ & $8(2,3,4)$ & $6(9,10,12)$ & $3(9,10,11)$ & $7(4,5,6)$ & $2(3,5,7)$ & $9(1,3,4)$ & $4(2,4,5)$ & $1(8,10,13)$ & $5(7,10,11)$ \\
\hline Job8 & $6(7,11,15)$ & $2(9,13,15)$ & $8(5,6,9)$ & $4(8,9,13)$ & $7(6,9,12)$ & $3(6,8,10)$ & $9(6,9,11)$ & $5(1,2,4)$ & $10(8,12,14)$ & $1(6,9,12)$ \\
\hline Job9 & $7(4,7,10)$ & $2(3,5,6)$ & $8(6,9,10)$ & $6(3,5,6)$ & $9(8,11,12)$ & $4(5,7,10)$ & $10(4,6,9)$ & $1(1,2,4)$ & $3(3,5,7)$ & $5(10,12,15)$ \\
\hline Job10 & $4(1,2,3)$ & $1(8,12,13)$ & $9(7,8,9)$ & $10(6,9,12)$ & $5(9,11,15)$ & $2(7,11,15)$ & $6(10,14,18)$ & $3(1,3,5)$ & $8(1,2,4)$ & $7(2,3,5)$ \\
\hline
\end{tabular}

Table 9 The experimental result for Case 5 in 10 run times

\begin{tabular}{|c|c|c|c|c|c|c|}
\hline \multirow[t]{2}{*}{ Run time } & \multicolumn{3}{|c|}{ Fuzzy Makespan } & \multicolumn{3}{|c|}{ Comparison index } \\
\hline & $\mathrm{s}_{1}$ & $\mathrm{~s}_{2}$ & $\mathrm{~s}_{3}$ & $\left(\mathrm{~s}_{1}+2 * \mathrm{~s}_{2}+\mathrm{s}_{3}\right) / 4$ & $\mathrm{~s}_{2}$ & $\mathrm{~s}_{3}-\mathrm{s}_{1}$ \\
\hline 1 & 31 & 47 & 63 & 47.00 & 47.00 & 32.00 \\
\hline 2 & 30 & 47 & 63 & 46.75 & 47.00 & 33.00 \\
\hline 3 & 30 & 47 & 63 & 46.75 & 47.00 & 33.00 \\
\hline 4 & 28 & 47 & 62 & 46.00 & 47.00 & 34.00 \\
\hline 5 & 29 & 47 & 63 & 46.50 & 47.00 & 34.00 \\
\hline 6 & 29 & 47 & 63 & 46.50 & 47.00 & 34.00 \\
\hline 7 & 27 & 47 & 62 & 45.75 & 47.00 & 35.00 \\
\hline 8 & 27 & 47 & 62 & 45.75 & 47.00 & 35.00 \\
\hline 9 & 27 & 47 & 62 & 45.75 & 47.00 & 35.00 \\
\hline 10 & 24 & 47 & 66 & 46.00 & 47.00 & 42.00 \\
\hline avg & 28.2 & 47 & 62.9 & 46.28 & 47.00 & 34.70 \\
\hline var & 4.18 & 0.00 & 1.43 & 0.23 & 0.00 & 7.57 \\
\hline
\end{tabular}

Table 11 The experimental result for Case7 in 10 run times

\begin{tabular}{llllllll}
\hline \multirow{2}{*}{ Run time } & \multicolumn{3}{l}{ Fuzzy Makespan } & & \multicolumn{3}{l}{ Comparison index } \\
\cline { 2 - 5 } \cline { 7 - 8 } & $\mathrm{s}_{1}$ & $\mathrm{~s}_{2}$ & $\mathrm{~s}_{3}$ & & $\left(\mathrm{~s}_{1}+2{ }^{2} \mathrm{~s}_{2}+\mathrm{s}_{3}\right) / 4$ & $\mathrm{~s}_{2}$ & $\mathrm{~s}_{3}-\mathrm{s}_{1}$ \\
\hline 1 & 99 & 129 & 152 & & 127.25 & 129.00 & 53.00 \\
2 & 97 & 128 & 154 & & 126.75 & 128.00 & 57.00 \\
3 & 93 & 121 & 155 & & 122.50 & 121.00 & 62.00 \\
4 & 96 & 127 & 160 & & 127.50 & 127.00 & 64.00 \\
5 & 92 & 119 & 160 & & 122.50 & 119.00 & 68.00 \\
6 & 92 & 119 & 160 & & 122.50 & 119.00 & 68.00 \\
7 & 95 & 125 & 164 & & 127.25 & 125.00 & 69.00 \\
8 & 95 & 125 & 164 & & 127.25 & 125.00 & 69.00 \\
9 & 94 & 128 & 163 & & 128.25 & 128.00 & 69.00 \\
10 & 92 & 126 & 165 & & 127.25 & 126.00 & 73.00 \\
avg & 94.50 & 124.70 & 159.70 & 125.90 & 124.70 & 65.20 \\
var & 5.61 & 14.01 & 21.12 & 5.64 & 14.01 & 38.62 \\
\hline
\end{tabular}




\begin{tabular}{|c|c|c|c|c|c|c|c|c|c|c|}
\hline \multirow{3}{*}{ M10 } & $\mathrm{O}[1,1]$ & $\mathrm{O}[8,3]$ & $\mathrm{O}[2,3]$ & $\mathrm{O}[7,5]$ & $\mathrm{O}[3,5]$ & $\mathrm{O}[6,8]$ & $\mathrm{O}[4,7]$ & $\mathrm{O}[9,7]$ & $\mathrm{O}[5,9]$ & $\mathrm{O}[0,10]$ \\
\hline & $(0,0,0)$ & $(4,7,9)$ & $(5,10,14)$ & $(9,14,18)$ & $(12,21,29)$ & $(14,25,34)$ & $(17,30,40)$ & $(20,34,45)$ & $(22,38,50)$ & $(24,42,55)$ \\
\hline & $(2,3,4)$ & $(5,10,14)$ & $(7,13,19)$ & $(10,17,22)$ & $(14,25,34)$ & $(17,30,40)$ & $(20,34,45)$ & $(22,38,50)$ & $(24,42,55)$ & $(26,45,59)$ \\
\hline \multirow{3}{*}{ M9 } & $\mathrm{O}[8,1]$ & $\mathrm{O}[2,2]$ & $\mathrm{O}[7,3]$ & $\mathrm{O}[6,5]$ & $\mathrm{O}[3,4]$ & $\mathrm{O}[4,6]$ & $\mathrm{O}[0,7]$ & $\mathrm{O}[5,8]$ & $\mathrm{O}[1,10]$ & $\mathrm{O}[9,10]$ \\
\hline & $(0,0,0)$ & $(3,4,5)$ & $(4,6,8)$ & $(8,14,19)$ & $(10,17,23)$ & $(13,24,34)$ & $(17,31,41)$ & $(19,34,46)$ & $(25,39,53)$ & $(26,44,58)$ \\
\hline & $(3,4,5)$ & $(4,6,8)$ & $(7,10,13)$ & $(10,17,23)$ & $(12,21,29)$ & $(15,27,38)$ & $(19,34,46)$ & $(20,36,49)$ & $(28,43,58)$ & $(27,46,62)$ \\
\hline \multirow{3}{*}{ M8 } & $\mathrm{O}[0,1]$ & $\mathrm{O}[3,3]$ & $\mathrm{O}[2,4]$ & $\mathrm{O}[1,5]$ & $\mathrm{O}[4,5]$ & $\mathrm{O}[5,6]$ & $\mathrm{O}[9,6]$ & $\mathrm{O}[8,7]$ & $\mathrm{O}[6,9]$ & $\mathrm{O}[7,10]$ \\
\hline & $(0,0,0)$ & $(6,11,14)$ & $(7,14,19)$ & $(8,16,23)$ & $(12,21,29)$ & $(14,26,35)$ & $(15,29,39)$ & $(18,33,44)$ & $(19,36,48)$ & $(22,41,54)$ \\
\hline & $(2,3,4)$ & $(7,14,19)$ & $(8,16,23)$ & $(12,21,29)$ & $(13,24,34)$ & $(15,29,39)$ & $(18,33,44)$ & $(19,36,48)$ & $(22,41,54)$ & $(24,44,59)$ \\
\hline \multirow{3}{*}{ M7 } & $\mathrm{O}[7,1]$ & $\mathrm{O}[1,2]$ & $\mathrm{O}[9,1]$ & $\mathrm{O}[4,2]$ & $\mathrm{O}[6,6]$ & $\mathrm{O}[2,6]$ & $\mathrm{O}[5,7]$ & $\mathrm{O}[3,7]$ & $\mathrm{O}[0,9]$ & $\mathrm{O}[8,10]$ \\
\hline & $(0,0,0)$ & $(3,4,5)$ & $(5,7,10)$ & $(7,11,15)$ & $(10,17,23)$ & $(13,21,28)$ & $(15,29,39)$ & $(18,33,44)$ & $(21,37,49)$ & $(26,41,56)$ \\
\hline & $(3,4,5)$ & $(5,7,10)$ & $(7,11,15)$ & $(8,14,19)$ & $(13,21,28)$ & $(14,24,32)$ & $(18,33,44)$ & $(21,37,49)$ & $(24,41,54)$ & $(29,45,61)$ \\
\hline \multirow{3}{*}{ M6 } & $\mathrm{O}[2,1]$ & $\mathrm{O}[0,2]$ & $\mathrm{O}[7,4]$ & $\mathrm{O}[1,4]$ & $\mathrm{O}[5,5]$ & $\mathrm{O}[8,6]$ & $\mathrm{O}[3,6]$ & $\mathrm{O}[4,8]$ & $\mathrm{O}[9,8]$ & $\mathrm{O}[6,10]$ \\
\hline & $(0,0,0)$ & $(2,4,5)$ & $(7,10,13)$ & $(9,14,18)$ & $(13,23,31)$ & $(14,26,35)$ & $(16,30,40)$ & $(20,34,45)$ & $(22,38,50)$ & $(25,42,55)$ \\
\hline & $(2,4,5)$ & $(5,9,11)$ & $(9,14,18)$ & $(10,16,21)$ & $(14,26,35)$ & $(16,30,40)$ & $(17,32,44)$ & $(21,37,49)$ & $(25,42,55)$ & $(26,44,58)$ \\
\hline \multirow{3}{*}{ M5 } & $\mathrm{O}[6,2]$ & $\mathrm{O}[3,2]$ & $\mathrm{O}[9,2]$ & $\mathrm{O}[0,3]$ & $\mathrm{O}[4,4]$ & $\mathrm{O}[5,4]$ & $\mathrm{O}[7,7]$ & $\mathrm{O}[2,9]$ & $\mathrm{O}[1,9]$ & $\mathrm{O}[8,9]$ \\
\hline & $(2,3,4)$ & $(3,7,9)$ & $(7,11,15)$ & $(8,13,18)$ & $(10,17,23)$ & $(11,19,26)$ & $(18,27,36)$ & $(21,32,44)$ & $(23,36,49)$ & $(25,39,53)$ \\
\hline & $(3,7,9)$ & $(6,11,14)$ & $(8,13,18)$ & $(10,17,23)$ & $(11,19,26)$ & $(13,23,31)$ & $(19,29,39)$ & $(23,36,49)$ & $(25,39,53)$ & $(26,41,56)$ \\
\hline \multirow{3}{*}{ M4 } & $\mathrm{O}[5,1]$ & $\mathrm{O}[8,2]$ & $\mathrm{O}[6,3]$ & $\mathrm{O}[1,3]$ & $\mathrm{O}[9,4]$ & $\mathrm{O}[2,7]$ & $\mathrm{O}[0,8]$ & $\mathrm{O}[7,9]$ & $\mathrm{O}[3,9]$ & $\mathrm{O}[4,10]$ \\
\hline & $(0,0,0)$ & $(3,4,5)$ & $(4,7,9)$ & $(5,10,14)$ & $(11,17,23)$ & $(14,24,32)$ & $(19,34,46)$ & $(20,36,49)$ & $(23,40,54)$ & $(25,44,58)$ \\
\hline & $(2,3,4)$ & $(4,7,9)$ & $(5,10,14)$ & $(7,14,19)$ & $(13,20,27)$ & $(15,27,37)$ & $(20,36,49)$ & $(23,40,54)$ & $(24,43,60)$ & $(26,47,62)$ \\
\hline \multirow{3}{*}{ M3 } & $\mathrm{O}[6,1]$ & $\mathrm{O}[5,3]$ & $\mathrm{O}[4,3]$ & $\mathrm{O}[8,5]$ & $\mathrm{O}[1,6]$ & $\mathrm{O}[0,6]$ & $\mathrm{O}[7,8]$ & $\mathrm{O}[2,10]$ & $\mathrm{O}[9,9]$ & $\mathrm{O}[3,10]$ \\
\hline & $(0,0,0)$ & $(4,6,8)$ & $(8,14,19)$ & $(9,17,23)$ & $(12,22,29)$ & $(14,26,35)$ & $(17,31,41)$ & $(23,36,49)$ & $(25,42,55)$ & $(26,44,58)$ \\
\hline & $(2,3,4)$ & $(5,8,11)$ & $(9,17,23)$ & $(12,22,29)$ & $(14,26,35)$ & $(17,31,41)$ & $(19,35,46)$ & $(24,39,54)$ & $(26,44,58)$ & $(27,47,62)$ \\
\hline \multirow{3}{*}{ M2 } & $\mathrm{O}[4,1]$ & $\mathrm{O}[5,2]$ & $\mathrm{O}[8,4]$ & O[9,3] & $\mathrm{O}[0,4]$ & $\mathrm{O}[6,7]$ & $\mathrm{O}[7,6]$ & $\mathrm{O}[1,7]$ & $\mathrm{O}[2,8]$ & $\mathrm{O}[3,8]$ \\
\hline & $(0,0,0)$ & $(2,3,4)$ & $(5,10,14)$ & $(8,13,18)$ & $(11,17,23)$ & $(15,22,29)$ & $(16,24,32)$ & $(18,27,36)$ & $(20,30,40)$ & $(21,37,49)$ \\
\hline & $(2,3,4)$ & $(4,6,8)$ & $(7,13,18)$ & $(11,17,23)$ & $(15,22,29)$ & $(16,24,32)$ & $(18,27,36)$ & $(20,30,40)$ & $(21,32,44)$ & $(22,40,54)$ \\
\hline \multirow{5}{*}{ M1 } & $\mathrm{O}[3,1]$ & $\mathrm{O}[7,2]$ & $\mathrm{O}[6,4]$ & $\mathrm{O}[2,5]$ & $\mathrm{O}[0,5]$ & $\mathrm{O}[9,5]$ & $\mathrm{O}[1,8]$ & $\mathrm{O}[8,8]$ & $\mathrm{O}[4,9]$ & $\mathrm{O}[5,10]$ \\
\hline & $(0,0,0)$ & $(3,4,5)$ & $(5,10,14)$ & $(8,16,23)$ & $(15,22,29)$ & $(16,24,32)$ & $(20,30,40)$ & $(19,36,48)$ & $(22,40,53)$ & $(25,44,58)$ \\
\hline & $(1,2,3)$ & $(4,6,8)$ & $(8,14,19)$ & $(11,21,29)$ & $(16,24,32)$ & $(17,26,35)$ & $(21,33,44)$ & $(22,40,53)$ & $(25,44,58)$ & $(26,47,62)$ \\
\hline & & & & \multirow[b]{2}{*}{0} & \multirow{2}{*}{\multicolumn{2}{|c|}{100}} & \multirow{2}{*}{\multicolumn{2}{|c|}{140}} & & \\
\hline & & & & & & & & & 60 & 80 \\
\hline
\end{tabular}

Fig. 4 Fuzzy Gantt chart of the best result for Case5 (fuzzy makespan=(27, 47, 62)) 


\begin{tabular}{|c|c|c|c|c|c|c|c|c|c|c|}
\hline \multirow{3}{*}{ M10 } & $\mathrm{O}[1,1]$ & $\mathrm{O}[6,1]$ & $\mathrm{O}[9,4]$ & $\mathrm{O}[5,7]$ & $\mathrm{O}[2,10]$ & $\mathrm{O}[0,10]$ & $\mathrm{O}[8,7]$ & $\mathrm{O}[7,9]$ & $\mathrm{O}[4,10]$ & $\mathrm{O}[3,10]$ \\
\hline & $(0,0,0)$ & $(10,11,14)$ & $(21,27,31)$ & $(38,47,63)$ & $(46,65,77)$ & $(61,76,103)$ & $(68,88,119)$ & $(73,95,127)$ & $(81,107,141)$ & $(86,111,149)$ \\
\hline & $(10,11,14)$ & $(20,22,28)$ & $(27,36,43)$ & $(42,54,71)$ & $(54,77,92)$ & $(70,88,116)$ & $(72,94,128)$ & $(81,107,141)$ & $(83,111,147)$ & $(92,119,160)$ \\
\hline \multirow{3}{*}{ M9 } & $\mathrm{O}[0,2]$ & $\mathrm{O}[9,3]$ & $\mathrm{O}[5,6]$ & $\mathrm{O}[2,9]$ & $\mathrm{O}[6,7]$ & $\mathrm{O}[4,7]$ & $\mathrm{O}[8,5]$ & $\mathrm{O}[7,7]$ & $\mathrm{O}[3,8]$ & $\mathrm{O}[1,10]$ \\
\hline & $(4,7,9)$ & $(14,19,22)$ & $(29,35,49)$ & $(37,52,63)$ & $(46,65,77)$ & $(47,68,81)$ & $(52,75,89)$ & $(66,84,112)$ & $(78,100,133)$ & $(80,103,138)$ \\
\hline & $(14,19,22)$ & $(21,27,31)$ & $(38,47,63)$ & $(46,65,77)$ & $(47,68,81)$ & $(52,75,89)$ & $(60,86,101)$ & $(72,93,123)$ & $(80,103,138)$ & $(90,117,153)$ \\
\hline \multirow{3}{*}{ M8 } & $\mathrm{O}[5,3]$ & $\mathrm{O}[6,2]$ & $\mathrm{O}[2,6]$ & $\mathrm{O}[7,3]$ & $\mathrm{O}[8,3]$ & $\mathrm{O}[1,5]$ & $\mathrm{O}[0,9]$ & $\mathrm{O}[3,5]$ & $\mathrm{O}[9,9]$ & $\mathrm{O}[4,9]$ \\
\hline & $(4,7,9)$ & $(20,22,28)$ & $(23,34,43)$ & $(30,44,53)$ & $(35,50,62)$ & $(46,58,77)$ & $(57,70,96)$ & $(64,82,111)$ & $(69,91,122)$ & $(70,93,126)$ \\
\hline & $(7,12,15)$ & $(22,25,32)$ & $(25,38,48)$ & $(35,50,62)$ & $(41,59,72)$ & $(48,62,83)$ & $(61,76,103)$ & $(68,87,117)$ & $(70,93,126)$ & $(73,97,132)$ \\
\hline \multirow{3}{*}{ M7 } & $\mathrm{O}[5,1]$ & $\mathrm{O}[0,1]$ & $\mathrm{O}[2,2]$ & $\mathrm{O}[4,2]$ & $\mathrm{O}[8,1]$ & $\mathrm{O}[6,5]$ & $\mathrm{O}[7,5]$ & $\mathrm{O}[3,4]$ & $\mathrm{O}[1,8]$ & $\mathrm{O}[9,10]$ \\
\hline & $(0,0,0)$ & $(1,3,4)$ & $(5,7,10)$ & $(7,10,15)$ & $(12,16,22)$ & $(40,45,55)$ & $(54,67,90)$ & $(61,77,104)$ & $(64,82,111)$ & $(72,93,125)$ \\
\hline & $(1,3,4)$ & $(4,7,9)$ & $(7,10,15)$ & $(12,16,22)$ & $(16,23,32)$ & $(44,50,61)$ & $(60,76,102)$ & $(64,82,111)$ & $(72,93,125)$ & $(74,96,130)$ \\
\hline \multirow{3}{*}{ M6 } & $\mathrm{O}[7,1]$ & $\mathrm{O}[6,3]$ & $\mathrm{O}[2,7]$ & $\mathrm{O}[3,2]$ & $\mathrm{O}[0,7]$ & $\mathrm{O}[8,4]$ & $\mathrm{O}[5,9]$ & $\mathrm{O}[9,7]$ & $\mathrm{O}[4,8]$ & $\mathrm{O}[1,9]$ \\
\hline & $(0,0,0)$ & $(22,25,32)$ & $(25,38,48)$ & $(29,43,55)$ & $(49,58,78)$ & $(53,65,88)$ & $(56,70,94)$ & $(58,74,99)$ & $(68,88,117)$ & $(72,93,125)$ \\
\hline & $(7,11,15)$ & $(31,35,44)$ & $(29,43,55)$ & $(33,48,63)$ & $(53,65,88)$ & $(56,70,94)$ & $(58,74,99)$ & $(68,88,117)$ & $(69,90,121)$ & $(78,102,135)$ \\
\hline \multirow{3}{*}{ M5 } & $\mathrm{O}[2,1]$ & $\mathrm{O}[3,1]$ & $\mathrm{O}[0,4]$ & $\mathrm{O}[1,3]$ & O[9,5] & $\mathrm{O}[4,6]$ & $\mathrm{O}[5,10]$ & $\mathrm{O}[7,8]$ & O[6,10] & O[8,10] \\
\hline & $(0,0,0)$ & $(5,7,10)$ & $(18,26,32)$ & $(24,35,45)$ & $(31,45,59)$ & $(49,57,68)$ & $(58,74,99)$ & $(72,93,123)$ & $(73,95,127)$ & $(80,105,138)$ \\
\hline & $(5,7,10)$ & $(10,15,20)$ & $(23,34,41)$ & $(31,45,59)$ & $(40,56,74)$ & $(54,63,77)$ & $(64,83,111)$ & $(73,95,127)$ & $(80,105,138)$ & $(90,117,153)$ \\
\hline \multirow{3}{*}{ M4 } & $\mathrm{O}[9,1]$ & $\mathrm{O}[2,3]$ & $\mathrm{O}[4,3]$ & $\mathrm{O}[5,5]$ & $\mathrm{O}[0,5]$ & $\mathrm{O}[1,4]$ & $\mathrm{O}[7,4]$ & $\mathrm{O}[3,3]$ & $\mathrm{O}[6,8]$ & $\mathrm{O}[8,6]$ \\
\hline & $(0,0,0)$ & $(7,10,15)$ & $(12,16,22)$ & $(21,26,36)$ & $(29,35,49)$ & $(39,47,65)$ & $(46,58,77)$ & $(54,67,90)$ & $(61,77,104)$ & $(63,81,109)$ \\
\hline & $(1,2,3)$ & $(8,13,19)$ & $(21,26,36)$ & $(29,35,49)$ & $(39,47,65)$ & $(46,58,77)$ & $(54,67,90)$ & $(61,77,104)$ & $(63,81,109)$ & $(68,88,119)$ \\
\hline \multirow{3}{*}{ M3 } & $\mathrm{O}[5,2]$ & $\mathrm{O}[2,4]$ & $\mathrm{O}[1,2]$ & $\mathrm{O}[6,4]$ & $\mathrm{O}[4,5]$ & $\mathrm{O}[0,8]$ & $\mathrm{O}[7,6]$ & $\mathrm{O}[9,8]$ & $\mathrm{O}[8,9]$ & $\mathrm{O}[3,9]$ \\
\hline & $(1,3,4)$ & $(8,13,19)$ & $(15,23,31)$ & $(31,35,44)$ & $(40,45,55)$ & $(53,65,88)$ & $(60,76,102)$ & $(68,88,117)$ & $(73,96,132)$ & $(80,103,138)$ \\
\hline & $(4,7,9)$ & $(15,23,31)$ & $(24,35,45)$ & $(40,45,55)$ & $(49,57,68)$ & $(57,70,96)$ & $(66,84,112)$ & $(69,91,122)$ & $(76,101,139)$ & $(86,111,149)$ \\
\hline \multirow{3}{*}{ M2 } & $\mathrm{O}[4,1]$ & $\mathrm{O}[5,4]$ & $\mathrm{O}[0,3]$ & $\mathrm{O}[8,2]$ & $\mathrm{O}[7,2]$ & $\mathrm{O}[2,8]$ & $\mathrm{O}[6,6]$ & $\mathrm{O}[9,6]$ & $\mathrm{O}[1,7]$ & $\mathrm{O}[3,6]$ \\
\hline & $(0,0,0)$ & $(7,12,15)$ & $(14,19,22)$ & $(18,26,32)$ & $(21,31,38)$ & $(30,44,53)$ & $(44,50,61)$ & $(40,56,74)$ & $(56,72,97)$ & $(68,87,117)$ \\
\hline & $(4,7,10)$ & $(12,19,23)$ & $(18,26,32)$ & $(21,31,38)$ & $(30,44,53)$ & $(37,52,63)$ & $(47,55,68)$ & $(47,67,89)$ & $(63,83,109)$ & $(76,97,129)$ \\
\hline \multirow{5}{*}{ M1 } & $\mathrm{O}[9,2]$ & $\mathrm{O}[2,5]$ & $\mathrm{O}[4,4]$ & $\mathrm{O}[0,6]$ & $\mathrm{O}[5,8]$ & $\mathrm{O}[1,6]$ & $\mathrm{O}[6,9]$ & $\mathrm{O}[8,8]$ & $\mathrm{O}[3,7]$ & $\mathrm{O}[7,10]$ \\
\hline & $(1,2,3)$ & $(15,23,31)$ & $(23,34,43)$ & $(39,47,65)$ & $(49,58,78)$ & $(48,62,83)$ & $(63,81,109)$ & $(72,94,128)$ & $(76,97,129)$ & $(81,107,141)$ \\
\hline & $(9,14,16)$ & $(23,34,43)$ & $(25,37,47)$ & $(49,58,78)$ & $(50,60,82)$ & $(56,72,97)$ & $(71,91,122)$ & $(73,96,132)$ & $(78,100,133)$ & $(87,116,153)$ \\
\hline & & & & & & & & & & \\
\hline & & & & & & & 20 & 40 & 60 & 80 \\
\hline
\end{tabular}

Fig. 5 Fuzzy Gantt chart of the best result for Case7 (fuzzy makespan=(92, 119, 160))

Table 13 The comparisons with other two algorithms

\begin{tabular}{|c|c|c|c|c|c|c|c|c|c|c|c|c|c|}
\hline & \multirow{2}{*}{$\begin{array}{l}\text { Problem } \\
\text { size }\end{array}$} & \multicolumn{4}{|l|}{ RKGA } & \multicolumn{4}{|l|}{ SMGA } & \multicolumn{4}{|l|}{ HDPSO } \\
\hline & & avg & $f_{1}$ & opt & $f_{1}$ & avg & $f_{1}$ & opt & $f_{1}$ & avg & $f_{1}$ & opt & $f_{1}$ \\
\hline Case1 & $6 \times 6$ & $28.9,36,43.1$ & 144.0 & $\begin{array}{c}29,36 \\
43\end{array}$ & 144.0 & $\begin{array}{l}28.2,36.1 \\
\quad 44.4\end{array}$ & 144.8 & $\begin{array}{l}29,36 \\
43\end{array}$ & 144.0 & $29,36,43$ & 144.0 & $\begin{array}{c}29,36 \\
43\end{array}$ & 144.0 \\
\hline Case2 & $6 \times 6$ & $56,80,103$ & 319.0 & $\begin{array}{c}56,80 \\
103\end{array}$ & 319.0 & $56,80,103$ & 319.0 & $\begin{array}{c}56,80 \\
103\end{array}$ & 319.0 & $56,80,103$ & 319.0 & $\begin{array}{c}56,80 \\
103\end{array}$ & 319.0 \\
\hline Case3 & $6 \times 6$ & $52.2,71,87.6$ & 281.8 & $\begin{array}{c}51,70 \\
86\end{array}$ & 277.0 & $\begin{array}{l}52.6,71.5 \\
88.5\end{array}$ & 284.1 & $\begin{array}{l}51,70, \\
86\end{array}$ & 277.0 & $51,70,86$ & 277.0 & $\begin{array}{c}51,70 \\
86\end{array}$ & 277.0 \\
\hline Case4 & $6 \times 6$ & $50,65,84$ & 264.0 & $\begin{array}{c}50,65 \\
84\end{array}$ & 264.0 & $50,65,84$ & 264.0 & $\begin{array}{l}50,65 \\
84\end{array}$ & 264.0 & $50,65,84$ & 264.0 & $\begin{array}{c}50,65 \\
84\end{array}$ & 264.0 \\
\hline Case 5 & $10 \times 10$ & $28.4,48,64.1$ & 188.5 & $\begin{array}{l}28,47 \\
62\end{array}$ & 184.0 & $\begin{array}{l}29.1,48.3 \\
64.5\end{array}$ & 190.2 & $\begin{array}{l}28,47 \\
66\end{array}$ & 188.0 & $28.2,47,62.9$ & 185.1 & $\begin{array}{c}27,47 \\
62\end{array}$ & 183.0 \\
\hline Case6 & $10 \times 10$ & $95.1,130.9,162.2$ & 519.1 & $\begin{array}{c}96,129 \\
60\end{array}$ & 414.0 & $\begin{array}{c}96.8,134.9 \\
164.7\end{array}$ & 531.3 & $\begin{array}{c}95,133, \\
161\end{array}$ & 522.0 & $\begin{array}{l}95.8,129.7 \\
160.5\end{array}$ & 515.7 & $\begin{array}{c}96,129 \\
60\end{array}$ & 414.0 \\
\hline Case 7 & $10 \times 10$ & $93,126.2,163.6$ & 509.0 & $\begin{array}{c}89,123 \\
158\end{array}$ & 493.0 & $\begin{array}{c}96.5,129.7 \\
168.3\end{array}$ & 524.2 & $\begin{array}{c}93,129, \\
168\end{array}$ & 519.0 & $\begin{array}{c}94.5,124.7 \\
159.7\end{array}$ & 503.6 & $\begin{array}{c}92,119 \\
160\end{array}$ & 490.0 \\
\hline Case 8 & $10 \times 10$ & $\begin{array}{l}84.6,115.9, \\
148.6\end{array}$ & 465.0 & $\begin{array}{c}85,116 \\
143\end{array}$ & 460.0 & $\begin{array}{c}86.1,118 \\
147.8\end{array}$ & 469.9 & $\begin{array}{c}88,115, \\
146\end{array}$ & 464.0 & $\begin{array}{c}86,115.7 \\
143.2\end{array}$ & 460.6 & $\begin{array}{c}85,116 \\
143\end{array}$ & 460.0 \\
\hline
\end{tabular}

$* f_{1}$ means $\left(s_{1}+2 * s_{2}+s_{3}\right)$ 